\title{
Moving from Pedagogy to Andragogy in Biomedical Engineering Design: Strategies for Lab-at-Home and Distance Learning
}

\author{
Philipp Gutruf (1), ${ }^{1}$ Urs Utzinger [D $^{2}{ }^{2}$ and Vignesh Subbian (iD ${ }^{3}$ \\ ${ }^{1}$ Department of Biomedical Engineering, Department of Electrical and Computer Engineering, BIO5 Institute, The University of \\ Arizona, Tucson, AZ, USA; ${ }^{2}$ Department of Biomedical Engineering, Department of Obstetrics and Gynecology, Department of \\ Electrical and Computer Engineering, BIO5 Institute, and College of Optical Sciences, The University of Arizona, Tucson, AZ, \\ USA; and ${ }^{3}$ Department of Biomedical Engineering, Department of Systems \& Industrial Engineering, BIO5 Institute, The \\ University of Arizona, Tucson, AZ, USA
}

(Received 30 June 2020; accepted 16 November 2020; published online 30 November 2020)

\begin{abstract}
Engineering design courses are particularly challenging to deliver in online or distance modalities because of the hands-on, collaborative nature of the design process and the need for physical resources and work spaces. In this work, we describe how we rapidly transformed two design courses in the middle two years of the biomedical engineering (BME) program to an online format during the 2019 coronavirus pandemic. In addition to time and safety constraints, we identified access to design spaces with biochemistry, computing, electronic, computing, and manufacturing tools, and team-based learning as major challenges to distance learning in BME design courses. To this end, we mapped and translated various course and design activities to an online environment using a combination of customized athome laboratory kits and distributed team structures. Drawing upon our pilot experience as well as principles from online and adult learning theories, we offer an overview of strategies to retain hands-on and team-based activities and rapidly implement BME design courses in online or distance modalities.
\end{abstract}

Keywords-Distance learning, Engineering design, Online education, Virtual teams.
Address correspondence to Vignesh Subbian, Department of Biomedical Engineering, Department of Systems \& Industrial Engineering, BIO5 Institute, The University of Arizona, Tucson, AZ, USA. Electronic mail: vsubbian@arizona.edu

Philipp Gutruf and Urs Utzinger are Joint First Authors.

\section{CHALLENGE STATEMENT}

Design is inherently a crucial and distinguishing engineering activity. ${ }^{3,5}$ It characterizes how engineers think and what they do: engineers "scope, generate, evaluate, and realize ideas". 9 Accordingly, engineering design education emphasizes (a) the ability to define problems through an iterative inquiry process; (b) recognize the systems context (i.e., systems thinking and systems design); (c) reason about and address uncertainties, (d) make design decisions, (e) function effectively as a part of team, and (f) communicate in multiple languages of design, including verbal, textual, graphical, computer-aided, and mathematical representations. ${ }^{4}$ While the majority of these skills may be achieved in distance or online modalities, engineering design courses are rarely offered in such modalities mainly because of the social process involved in teambased activities as well as access to physical resources such as laboratory spaces and manufacturing equipment. The 2019 coronavirus disease (COVID-19) pandemic challenged the status quo and forced all courses, including design courses, to be moved to distance or online modalities.

The undergraduate Biomedical Engineering (BME) curriculum at the University of Arizona includes a sequence of four design courses from freshman to senior year. Of these, the design courses in the middle two years-Sophomore Intermediate Design (BME 210) and Medical Device Design (BME 310) - are specific to BME. In the sophomore design course, students learn general concepts of mechanical, elec- 
tronic, and software design and engage in the creation and control of electromechanical systems (e.g., robotic arm to activate switches). The course culminates in a design competition where students demonstrate their electromechanical system for a given set of constraints. In the junior design course, students design medical devices using wireless electronics, design and simulation software, microfluidics, and biochemical analysis. The course culminates in a team-defined medical device challenge where students demonstrate the design, implementation, and testing of their device.

The specific challenge during the COVID-19 university closure was teaching and learning in the two BME design courses, both of which involve hands-on and team-based experiences with a focus on developing contemporary engineering skills such as computer aided design (CAD) and simulation, 2D and 3D manufacturing, data analysis, and integrated software development. First, access to design spaces, expensive manufacturing equipment, and hardware resources were no longer feasible because of stay-at-home orders and physical distancing restrictions. Second, teambased learning as well as personalized support from course staff to student teams were also challenging, given that several students had to change their living arrangements multiple times during the semester, among various other reasons. We present how the two design courses were rapidly transformed to enable distance learning during Spring 2020 semester and provide instructional and administrative strategies that can be readily adapted for similar design courses in the near-term (2020-2021 academic year) and beyond.

\section{NOVEL INITIATIVE}

For the sophomore design course, the team-based approach and in-person collaborative nature of the design courses were changed to an individual, lab-athome approach. We created a custom laboratory kit within a two-week time frame and shipped the kit to each student's mailing address at that time. The kit included a pre-configured, single-board computer and hardware components needed for various design activities, including the design competition. A detailed description of learning modules, materials used in this course, estimated costs, and rationale are included as supplemental material in Appendix A.

For the medical device design course, the design process was upheld to the extent possible with few exceptions. Students created and submitted custom 3D CAD designs of medical devices on their personal computers. The fabrication elements of the design that could not be executed by the students in person were demonstrated live via videoconferencing $\left(\right.$ Zoom $\left.^{\circledR}\right)$ and resulting parts where shipped to students for testing and experimentation. Students then developed study protocols to test the device (epifluidic sweat sensor) on themselves at home. For the competition at the end of the course, students worked in virtual teams to define and design a medical device. Teams were provided a catalog of integrated components and sensors that they could select from. As a part of their team and project management plan, students self-organized their team workload distribution and selected components and microcontrollers, which were then shipped for experimentation and firmware design, while mechanical design, electronics layout, and documentation were completed digitally. A description of learning modules, hardware and software materials used in the course, and exemplar designs produced by students are described in Appendix $B$.

In addition to the course design details and at-home laboratory kits provided in appendices, we summarize and highlight the following strategies that design educators can readily adapt and extend for their courses in the immediate near future:

- Create a centralized digital environment that will serve as means for documentation, submission, review, and version control of design assets: The medical device design course used a suite of Google for Education applications to develop and maintain a website with both publicly-accessible information (course organization, learning modules, design primers) ${ }^{7}$ and private features (laboratory sign-in and sign-out system, submission system for design assets). The submission system, developed based on Google Forms and Google Drive, allowed for uploading of design files (e.g., .STL for 3D printing and .DXF for laser cutting), along with metadata necessary for review and version control (time, design version, author etc.). For advanced design courses, a more robust version control system such as GitHub ${ }^{\circledR}$ with interactive capabilities (e.g., viewing 3D designs and differences between design versions) ${ }^{10}$ may be considered.

- Foster virtual, distributed team work through welldefined processes for accountability and collaboration: For effective virtual collaboration on design activities, it is essential for each team to develop a "contract", documenting strengths and expertise of each team member, joint and individual responsibilities, hardware components selected for the design project, shipping address, design milestones, and team operational plan. This contract can also be used for formative assessment of team activities. Additionally, remote pair programming and 
designing can be encouraged, where team members meet virtually and alternate between driver and navigator roles - the driver performs a design activity (assembly, CAD, firmware programming etc.) and the navigator observes and reviews each step of the activity). While certain teams in medical device design course adopted variants of pair programming and designing for their team work, this strategy was not explicitly enforced and assessed in our design courses and is an area of future work using evidence from software engineering literature. ${ }^{6,11}$

- Adopt low stakes formative assessment throughout the design cycle: The modular and iterative nature of the design process allows for a series of low stakes assessment starting with development of a team contract, followed by two intermediate design reviews, and ending with development and execution of test plans. For virtual team work assessment, it is critical to require student teams to develop a detailed operational plan for pair programming and designing and submit a reflection summarizing their role (driver vs. navigator), time spent, type of design activity, and overall experience for each paired virtual session.

\section{REFLECTION}

Despite the virtual and isolated format of the design courses, students were still able to engage in the entire design cycle from problem definition and design to testing and validation. While some students continued to work together virtually, the social process of working and learning in a design team suffered the most. The medical device design course did, however, retain some of the team activities and emphasized documentation of teams activities and virtual presentation of the designs (e.g., see Appendix B, 3D photorealistic rendering tutorial).

Prior to the pandemic, our department had just completed offering the first fully online BME course. ${ }^{12}$ This course was designed using Anderson's model for online learning that highlights four attributes for interactions between student(s), content (knowledge), and the educator: self-directed learning (student-content), structured learning resources (educator-content), collaborative learning (student-student), and a community of inquiry (student-educator). ${ }^{2,1}$ The course also leverages Hackman's team effectiveness model from organizational psychology ${ }^{8}$ to inform team-based activities as well as nurture andragogical principles. Given that working in distributed teams will become even more prevalent in engineering practice post
COVID-19, the experience and approaches from the existing online course will need to be translated to foster a virtual team-based experience in design courses. For example, a well-structured and interactive digital environment is needed to enable effective communication within and across design teams as well as collaborative decision-making and problem-solving among team members. Such an environment may also include a more automated submission and version control system to streamline management of design revisions. While we used Google for Education (G Suite) as the preferred environment, Microsoft Teams ${ }^{\circledR}$ and Open $E d X^{\circledR}$ are potential alternatives for design courses. Institutionally-supported learning management systems may also be considered, provided they offer seamless management of design assets as well as facilitate effective interactions between the students, educator(s), and content.

In the medical device design course, the protocol development for at-home studies transported valuable insight for students in terms of device testing as well as limitations and strengths of their design. It was also noted that live demonstrations of fabrication of student designs was lengthy and largely appealing only to students that conceived the design and less so for the others. In the next virtual offering, we plan to modularize the demonstrations into smaller sessions (e.g., demo modules for each fabrication step) and move demos to either offline or to individual teams.

In terms of hardware resources, the logistics of creating and shipping 40 + laboratory kits was significant, especially in an environment where University resources were shut down, and where the supply chain for parts started to slow down. Several additional shipments were needed to mitigate issues of missing or incompatible parts and lost packages. With more time and preparation ahead of the start of the term, such issues could be averted.

Overall, we estimate that the additional effort by a single instructor to transform a course into an online or distance format is approximately $80 \mathrm{~h}$, excluding regular teaching activity and support from teaching assistants. Based on our collective experience in Spring 2020, we offer a visual summary of activities (Fig. 1) in BME design courses that can be translated to online or distance modalities. In addition to at-home lab kits described in the appendices, a combination of the three strategies - a seamless online environment for managing design assets, processes for distributed team work and collaboration, and a robust set of low stakes formative assessment - are necessary for effectively offering design courses in an online or distance modalities. 
Design Documentation and Presentation

Software and firmware development

Mechanical design and fabrication

Device testing and validation

Teamwork and collaboration

Biochemical experimentation requiring on-campus facilities
Shared digital notebook, 3D photorealistic rendering using CAD

At-home lab kit including single board computers and

microcontrollers shipped to students

CAD design at home and send to course staff for fabrication in a live

or asynchronous demonstration

Co-develop and execute test plans at home to evaluate the design

Provide goals and guidelines for virtual interaction within teams (e.g., pair programming and designing)

Not feasible at home. With aggressive safety measures (staggered schedules, physical distancing, regular cleaning etc.), teams may be allowed to use on-campus design spaces.

Minimal effort
High effort

FIGURE 1. Summary of strategies for BME design courses in distance or online modalities.

\section{ELECTRONIC SUPPLEMENTARY MATERIAL}

The online version of this article (https://doi.org/10. 1007/s43683-020-00043-8) contains supplementary material, which is available to authorized users.

\section{AUTHOR CONTRIBUTIONS}

As a team, the authors are actively engaged in advancing the educational mission of the BME department at the University of Arizona. Dr. UU created the sophomore design course in Spring 2017. Dr. PG developed learning modules for the junior design course and together with Dr. UU taught the course for the first time in Spring 2020. Drs. UU and PG also created the college's medical device design laboratory for undergraduate students. Dr. VS initiated online education efforts for the BME department and converted two BME courses to a fully online format, one in Fall 2019 and another in Spring 2020. All authors contributed the development of this manuscript.

\section{AVAILABILITY OF DATA AND MATERIAL}

A detailed description of course materials for both BME design courses are provided as supplemental material.

\section{FUNDING}

A philanthropic gift from Peter and Nancy Salter supported the creation of the medical device design course described in this work as well as the construction of a medical device design laboratory, which is a physical learning and collaboration space for the course.

\section{CONFLICTS OF INTEREST}

None to report.

\section{CONSENT FOR PUBLICATION}

Permissions were obtained from those students whose work are provided as examples in the supplemental material.

\section{REFERENCES}

${ }^{1}$ Anderson T. Towards a theory of online learning. Theory Pract Online Learn. 2004;2:109-19.

${ }^{2}$ Anderson T. Theories for learning with emerging technologies. Emerg Technol Distance Educ. 2010. https://doi. org/10.15215/aupress/9781771991490.01.

${ }^{3}$ Dym CL. Design, systems, and engineering education. Int $\mathrm{J}$ Eng Educ. 2004;20:305-12.

${ }^{4}$ Dym CL, Agogino A, Eris O, Frey DD, Leifer LJ. Engineering design thinking, teaching, and learning. J Eng 
Educ. 2005;94:103-20. https://doi.org/10.1109/EMR.2006. 1679078

${ }^{5}$ Evans DL, McNeill B, Beakley G. Design in engineering education: past views of future directions. J Eng Educ. 1990;79:517-22.

${ }^{6}$ Flor NV. Globally distributed software development and pair programming. Commun ACM. 2006;49:57-8. http s://doi.org/10.1145/1164394.1164421.

${ }^{7}$ Gutruf P, Utzinger U. Medical Device Design Lab. https:// sites.google.com/email.arizona.edu/bme310/. Accessed 29 July 2020.

${ }^{8}$ Hackman JR. The design of work teams. In: Lorsch J, editor. Handbook of organizational behavior. Englewood Cliffs: Prentice-Hall; 1987.

${ }^{9}$ Sheppard SD. A description of engineering: an essential backdrop for interpreting engineering education. Claremont: Mudd Design Workshop IV. Harvey Mudd College; 2003.
${ }^{10}$ Skalnik M. 3D file diffs - the GitHub blog. 17 Sep 2013. h ttps://github.blog/2013-09-17-3d-file-diffs/. Accessed 29 July 2020

${ }^{11}$ Subbian V, Purdy C. A hybrid design methodology for an introductory software engineering course with integrated mobile application development. 121st annual ASEE conference. 2014.

${ }^{12}$ Subbian V, Whitaker DB. Switching modalities: implications of online education in biomedical engineering. American Society for Engineering Education (ASEE) annual conference \& exposition. 2020.

Publisher's Note Springer Nature remains neutral with regard to jurisdictional claims in published maps and institutional affiliations. 